Review

\title{
Seipin Deficiency as a Model of Severe Adipocyte Dysfunction: Lessons from Rodent Models and Teaching for Human Disease
}

\author{
Jocelyne Magré and Xavier Prieur *(1)
}

Citation: Magré, J.; Prieur, X. Seipin Deficiency as a Model of Severe Adipocyte Dysfunction: Lessons from Rodent Models and Teaching for Human Disease. Int. J. Mol. Sci. 2022, 23, 740. https://doi.org/ $10.3390 /$ ijms 23020740

Academic Editor: Jean-françois Tanti

Received: 15 December 2021

Accepted: 7 January 2022

Published: 11 January 2022

Publisher's Note: MDPI stays neutral with regard to jurisdictional claims in published maps and institutional affiliations.

Copyright: (C) 2022 by the authors. Licensee MDPI, Basel, Switzerland. This article is an open access article distributed under the terms and conditions of the Creative Commons Attribution (CC BY) license (https:// creativecommons.org/licenses/by/ $4.0 /)$.

\begin{abstract}
Centre National de la Recherche Scientifique, Institut National de la Santé et de la Recherche Médicale, L'institut du Thorax, Université de Nantes, F-44000 Nantes, France; jocelyne.magre@univ-nantes.fr

* Correspondence: Xavier.prieur@univ-nantes.fr
\end{abstract}

\begin{abstract}
Obesity prevalence is increasing worldwide, leading to cardiometabolic morbidities. Adipocyte dysfunction, impairing white adipose tissue (WAT) expandability and metabolic flexibility, is central in the development of obesity-related metabolic complications. Rare syndromes of lipodystrophy characterized by an extreme paucity of functional adipose tissue should be considered as primary adipocyte dysfunction diseases. Berardinelli-Seip congenital lipodystrophy (BSCL) is the most severe form with a near absence of WAT associated with cardiometabolic complications such as insulin resistance, liver steatosis, dyslipidemia, and cardiomyopathy. Twenty years ago, mutations in the BSCL2 gene have been identified as the cause of BSCL in human. BSCL2 encodes seipin, an endoplasmic reticulum (ER) anchored protein whose function was unknown back then. Studies of seipin knockout mice or rats demonstrated how seipin deficiency leads to severe lipodystrophy and to cardiometabolic complications. At the cellular levels, seipin is organized in multimers that are particularly enriched at ER/lipid droplet and ER/mitochondria contact sites. Seipin deficiency impairs both adipocyte differentiation and mature adipocyte maintenance. Experiments using adipose tissue transplantation in seipin knockout mice and tissue-specific deletion of seipin have provided a large body of evidence that liver steatosis, cardiomyopathy, and renal injury, classical diabetic complications, are all consequences of lipodystrophy. Rare adipocyte dysfunctions such as in BSCL are the key paradigm to unravel the pathways that control adipocyte homeostasis. The knowledge gathered through the study of these pathologies may bring new strategies to maintain and improve adipose tissue expandability.
\end{abstract}

Keywords: adipocyte; lipodystrophy; lipotoxicity; insulin resistance; seipin; adipocyte dysfunction; diabetic complications

\section{Introduction}

Obesity prevalence increases worldwide, leading to cardiometabolic morbidities [1] The adipose tissue expandability hypothesis suggests that when adipose tissue reaches its personal threshold of expansion, additional lipids cannot be stored properly in adipocytes [2]. Instead, lipids deposit ectopically in various organs such as the liver, muscle, pancreas, heart, and vessels, where they activate lipotoxic pathways that compromise the function of these organs and contribute to insulin resistance [3]. Growing evidence supports that adipocyte dysfunction, which impairs adipose tissue expandability and plasticity, is central in the development of obesity-related metabolic complications [4]. Genetic studies revealed the existence of an allelic signature associated with a favorable adiposity pattern: high subcutaneous storage capacity, even associated with elevated BMI, lowers the risk of T2D and heart disease [5].

Conversely, rare syndromes of lipodystrophy characterized by an extreme paucity of functional adipose tissue are associated with severe cardiometabolic complications [6,7]. These are primary adipocyte dysfunction disorders, and the most severe is congenital generalized lipodystrophy (CGL), which is also named Berardinelli-Seip congenital lipodystrophy (BSCL) [8]. BSCL patients display a near absence of adipose tissue from birth or early 
infancy, affecting all metabolically fat pads (subcutaneous and visceral fat) and the early development of metabolic complications. Patients are recognized due to the lack of body fat, the visible veins, and prominent muscularity that cause a severe and striking phenotype. Patients with BSCL develop insulin resistance and associated cardiometabolic abnormalities soon after birth or in childhood. These include hyperinsulinemia and glucose intolerance to overt diabetes hepato-steatosis, cardiomyopathy, and hypertriglyceridemia [8]. This disease was reported in the 1950s in Brazil by Dr. Waldemar Berardinelli [9] and in Norway by Dr. Martin Seip [10]. Since then, $\approx 300 / 500$ patients with BSCL have been reported in the literature with an estimated prevalence of $\approx 0.1$ to 5 persons per million depending on the population.

Four subtypes are currently distinguished according to the causative gene involved-BSCL1 due to mutations in the gene encoding the enzyme 1-acylglycerol-3-phosphate O-acyltransferase 2 (AGPAT2) [11], BSCL2 due to mutations in the gene encoding seipin [12], BSCL3 and BSCL4 due to mutations in caveolae-associated proteins, respectively caveolin-1 (CAV1) [13] and PTRF (Polymerase 1 and Transcription Release Factor), also named CAVIN1 [14]. The first two subtypes linked to AGPAT2 or Seipin are, by far, the most frequent, representing about 95\% of the patients [15-17].

Phenotypic differences have been observed among the known BSCL genotypes with those carrying BSCL2 alterations displaying the most extreme phenotype. These patients have almost no detectable adipose tissue in any depots. In addition to the lack of the metabolically active adipose tissue, they lack the mechanical fat usually present in retroorbital, palm, sole, and periarticular regions. They are at much higher risk for hypertrophic cardiomyopathy and have metabolic complications that are particularly severe with an earlier onset of diabetes $[18,19]$. BSCL2 patients often present with mild mental retardation and in a few cases with progressive encephalopathy with a poor prognostic during childhood [19-21].

The mechanism of adipose tissue loss is specific to each BSCL subtype depending on the biological function of the mutated gene [22]. Twenty years ago, when BSCL2 has been identified, seipin function was unknown. In silico studies could not predict any functional domain in seipin sequence [12]. Cellular studies revealed that seipin is an ER-anchored protein [23,24] and that seipin deficiency alters the lipid droplets (LD) morphology [23-26]. Seipin consists of a conserved loop located in the lumen of the ER, two transmembrane domains (amino acids 27-47 and 247-270), and the $\mathrm{N}$ - and C-terminal ends exposed in the cytosol [27,28]. Biochemistry studies [29], atomic force microscopy, and recently cryoelectron microscopy $[30,31]$ have shown that seipin forms oligomers, either dodecamers or undecamers depending on species and cell type, to form a ringlike/donut structure of homo-oligomers with the transmembrane domains at the periphery and hydrophobic helices positioned at the inner surface of the ring, protruding into the luminal leaflet of the ER bilayer [30-32]. The luminal domain of each Seipin monomer adopts a similar fold with an eight-strand $\beta$-sandwich typical of certain lipid-binding proteins [30]. Recently, it was further shown that the hydrophobic helices contain serine residues that interact directly with TAG within the membrane. They concentrate TAG molecules, thereby facilitating lens formation and LD budding [33,34].

In addition, seipin is needed to ensure full ability of pre-adipocytes to differentiate into mature adipocytes $[35,36]$. However, it was unknown how seipin deficiency could lead to lipodystrophy, and more generally, how lipodystrophy leads to cardiometabolic complications was poorly understood. Thus, animal models of these conditions are very useful tools to study the function of the genes involved, to characterize the pathophysiology of these extreme cases of primary adipocyte dysfunction, and to test promising therapies. In this review, starting from the adipose tissue phenotype to the different cardiometabolic complications, we will discuss how the use of different rodent models, with total and tissue-specific deletions of seipin, largely increased our knowledge of the pathophysiology of BSCL. 


\section{Seipin Deficiency Leads to Severe Lipodystrophy}

\subsection{Adipose Tissue Loss}

The total deletion of seipin in mice (seipin knockout, SKO, mice) leads to a dramatic loss of white adipose tissue (WAT) assessed by magnetic resonance imaging (MRI) and $X$-ray analysis and confirmed by dissection [37-39] in 8 to 12-week-old males and females. The residual adipose tissue represents less than $10 \%$ of the adipose tissue measured in WT littermates for the inguinal and mesenteric WAT [38], but gonadal WAT was completely absent. Similarly, 20-week-old SKO rats are severely lipodystrophic and display 95\% adipose tissue loss [40]. In all models, leptin levels were 40 to $30 \%$ decreased, and adiponectin plasma concentrations were dramatically reduced, reaching a $90 \%$ decrease in 8 to 12-week-old mice [37-39]. Despite the consistent decrease in leptin levels, food intake was increased modestly in some reports [38,39] but unchanged in the other publications [37]. The gene expression profiling of WAT showed a genuine alteration with a strong reduction in the mRNA levels of key adipocyte markers such as perilipin1 (Plin1), peroxisome proliferator-activated receptor gamma (Pparg), adipocyte protein 2 (Ap2), and hormone-sensitive lipase $(\mathrm{Hsl})$.

In young mice, we reported the presence of residual gonadal adipose tissue, and we described a loss of all WAT fat pads from 4 to 14-week-old mice along with a reduction in adiponectin levels [41]. This observation suggested that lipodystrophy might arise at least partially from a loss of mature adipocytes. Consistently, under the adipocyte specificpromoter of the AdipoQ gene, CRE-mediated Bscl2 deletion leads to early (6 to 12 weeks of age) and severe lipodystrophy with a massive loss of gonadal and subcutaneous WAT and a $80 \%$ decrease in circulating adiponectin [42]. Under the AP2 promoter [43], seipin deletion leads to a less severe and more progressive adipose tissue loss, but the efficiency of the AP2 CRE has been challenged [44] and might explain this mild lipodystrophic phenotype. Intriguingly, in both models, in contrast to adiponectin, leptin levels were not altered, suggesting that the remaining adipose tissue was sufficient to maintain a critical production of the satiety adipokine $[42,43]$. Food intake was not increased. Finally, tamoxifen-inducible seipin deletion under the ERT2-AdipoQ promoter also induces a progressive loss of adipose tissue, strongly supporting that seipin is required for mature adipocyte maintenance $[45,46]$.

\subsection{Adipogenesis Impairment}

Whether lipodystrophy is a developmental issue or an impairment of mature adipocyte maintenance remains unknown. Early reports demonstrated that seipin deficiency in mesenchymal stem cell lines C3H10T1/2 [35], in 3T3-L1 pre-adipocytes [36], and in mouse embryonic fibroblasts (MEF) [38] prevents normal adipogenesis. Notably, the induction of PPAR $\gamma$ target genes mRNA levels during adipogenesis is impaired in the absence of seipin, and thiazolidinediones (TZD), the potent PPAR $\gamma$ agonists, improve the ability of seipin-deficient cells to differentiate into adipocytes in vitro [36,38]. This suggests that seipin deficiency somehow blunts PPAR $\gamma$ activation during adipogenesis. Importantly, in SKO [38] and adipocyte-deficient seipin mice [43], TZD treatment increases adipose tissue mass and improves the metabolic phenotype of these animals. A clinical case study even reported an improvement of $\mathrm{HbA} 1 \mathrm{C}$ and the presence of little subcutaneous WAT in a BSCL2 patient after 5 years of pioglitazone treatment [47].

At the mechanistic level, how seipin might regulate adipogenesis and PPAR $\gamma$ activity has been extensively studied. Evidence from yeast highlighted that seipin deficiency leads to abnormal accumulation of phosphatidic acid (PA) [25]. It has been then suggested that PA accumulation could inhibit PPAR $\gamma$ activity [48]. Importantly, PA is increased in seipin deficient adipose tissue in vivo [43]. Using 3T3-L1 preadipocytes, Yang's lab demonstrated that seipin interacts with the glycerol-3-phosphate acyltransferase 3 (GPAT3) and inhibits its activity (Figure 1). They further demonstrated that GPAT3 inhibition improves adipogenesis in SKO MEF [49]. Finally, in vivo, the double KO mice for seipin and GPAT3 display an improvement in WAT properties and systemic insulin sensitivity as well as a reduction in liver steatosis [50]. GPAT3 catalyzes the synthesis of lysophosphatidic acid (LPA) that is 
transformed into PA by the LPA-acyltransferase- $\$$, AGPAT2; then, Lipin1, a phosphatidate phosphatase enzyme, allows the synthesis of DAG. Rochford's Lab [32,51] demonstrated that Seipin oligomer interacts with AGPAT2 and Lipin 1, and they proposed that these physical interactions might be particularly relevant during adipocyte differentiation as both Lipin1 [52] and AGPAT2 [53,54] are adipogenesis regulators (Figure 1). They further demonstrated that seipin negatively regulates PA levels [51]. They also confirmed the physical interactions between seipin and GPAT3 but did not confirm that GPAT3 inhibition restores the adipogenic properties of seipin deficient pre-adipocytes. Altogether, seipin oligomers physically scaffold three key enzymes of the glycerophospholipid and triglyceride synthetic pathway, and these interactions are relevant during adipogenesis, at least in vitro. Further studies would be needed to specifically unravel how and when seipin oligomer orchestrates TAG synthesis during the adipogenic process.

A

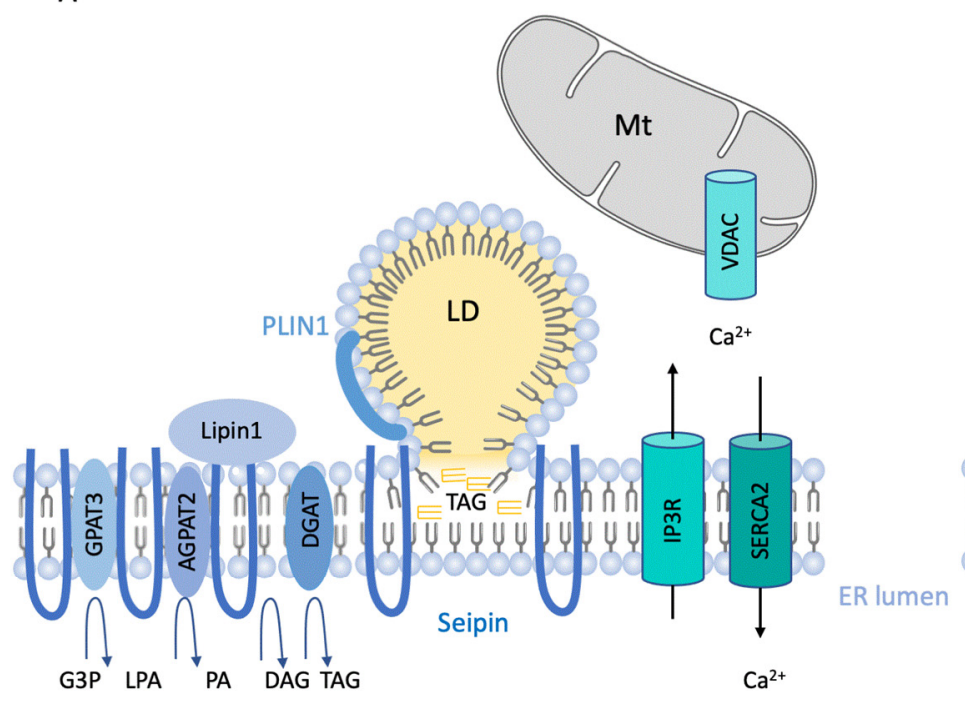

B

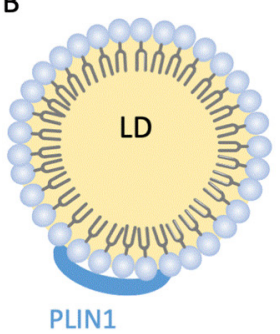

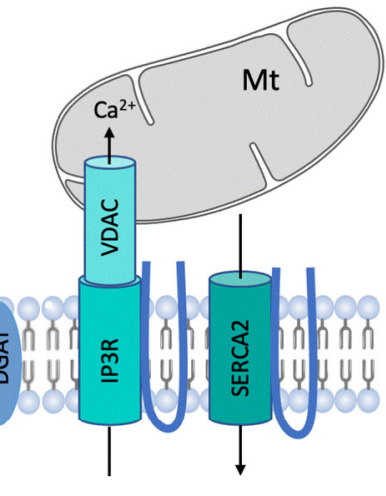

$\mathrm{Ca}^{2+}$

$\mathrm{Ca}^{2+}$

Figure 1. Seipin function in mature adipocytes. Seipin is an ER-anchored protein organized as oligomers. When cells are lipid loaded (A), seipin is enriched at the endoplasmic reticulum (ER)/lipid droplet (LD) contact sites and has been shown to be crucial in triglycerides (TAG) flow from the ER to the LD. Seipin interacts with Perilipin1 and with several TAG synthesis enzymes such as glycerol3-phosphate acyltransferase (GPAT3), 1-acyl-sn-glycerol-3-phosphate acyltransferase beta (AGPAT2), and LIPIN. No interaction with diacylglycerol acyltransferases (DGAT) has been formally reported. (B)-In the fasting state, seipin is enriched at the ER/mitochondria (Mt) contact sites, also named mitochondria-associated membranes (MAM), and participates in ER/mitochondria calcium $\left(\mathrm{Ca}^{2+}\right)$ flux and mitochondrial activity. Seipin is in close proximity to MAM Ca ${ }^{2+}$ regulators IP3(inositol 1,4,5-trisphosphate) receptor (IP3R), voltage-dependent anion channel (VDAC), and sarco-/ER Ca ${ }^{2+}$ ATPase 2 (SERCA2) as well as glycerol-3-phosphate (G3P), lysophosphatidic acid (LPA), phosphatidic acid (PA), and diacylglycerol (DAG).

\subsection{Accelerated Lipolysis}

Studying the differentiation of MEF isolated from SKO embryos, Chen et al. were the first to highlight an increase in basal lipolysis. The glycerol and NEFA release were elevated at days 4 to 6 of differentiation, and this was associated with a chronic activation of the protein kinase A (PKA) pathway, resulting in an increased phosphorylation of HSL and PLIN1 [39]. The pharmacological inhibition of lipase activity increased TAG storage in SKO MEF, suggesting that elevated basal lipolysis was, at least partially, responsible for adipogenesis impairment. This elevated lipolysis during MEF differentiation has been reproduced in another report [38]. Ex vivo, in SKO WAT explants, Chen et al. reported an increase in basal lipolysis [39], whereas we observed a decrease in basal and stimulated lipolysis [41]. As in vitro, lipolysis activation was maximal at day 4 to 6 of differentiation and was similar 
to control cells at days 8; we could speculate that the phenomenon is transitory given that at a certain stage, the residual adipose tissue is nearly devoid of full adipocytes. Aiming to test the relevance of this observation in vivo, Chen's lab crossed SKO mice with adipose triglyceride lipase (ATGL) KO mice and demonstrated that ATGL ablation rescues the lipodystrophic phenotype and the metabolic complications associated with seipin deficiency [55]. ATGL ablation also improves the adipocyte differentiation of seipin-deficient MEF in vitro [55]. Finally, inducible deletion of seipin in developing adipocytes leads to an elevated NEFA release in WAT explants, which is associated with chronic activation of the PKA pathway [45]. Together with the increased NEFA release, several studies reported an increase in FA oxidation and thermogenesis $[45,56]$ in seipin-deficient WAT. Recently, using an inducible deletion of seipin, we reported that this induction of FA catabolism was transitory, suggesting that it may be an early event accounting for the loss of TAG store in seipin-deficient adipose tissue [46]. Altogether, several pieces of evidence support that abnormally elevated NEFA release and catabolism might contribute to the lipodystrophic phenotype of seipin-deficient mice.

\subsection{The Lipid Droplet Safe Guard}

Early studies in yeast have shown that seipin localizes at ER/LD contact sites [23] and that seipin deficiency alters LD morphology in yeast [23-25] and human cells [26]. Seipin is required for the normal rate of LD formation [57] and is necessary for converting nascent to mature LD [58]. It has been further reported that seipin acts as an ER-to-LD targeting protein during LD maturation, recruiting lipids and proteins in a specific, stepwise, and timeregulated manner [59]. Consistently, in BSCL2 patients' fibroblasts, ER/LD contacts appear abnormal and LD show aberrant mobility. This implication of seipin in orchestrating the ERto-LD recruitment of lipids and proteins was recently confirmed in yeast [60]. Importantly, when seipin is trapped at cytosolic side of the nuclear envelope, it promotes the formation of LD at this site specifically. Finally, it has been demonstrated that seipin interacts with TAG and DAG and helps their flow to the LD by maintaining functional ER-LD contacts and counteracting LD ripening [33,34,61]. Indeed, seipin seems to rearrange the ER membrane at specific ER domains favoring LD biogenesis and maturation [34]. Altogether, a large body of work performed in cellular systems supports that seipin oligomers organize the ER membrane subdomains to promote normal LD assembly and to prevent TAG ripening (see review [62], Figure 1A). Although this has not been formally demonstrated during adipogenesis, it is highly likely that the lack of seipin prevents normal LD in mature adipocytes. In addition, as seipin binds TAG and DAG and targets them to the LD, we may hypothesize that in the absence of seipin, TAG and DAG would be misaddressed and therefore could be more easily the target of lipases. In addition, as suggested by Rao and Goodman, small and abnormal LDs could be the substrate for lipophagy that would result in unstimulated lipolysis [62]. Therefore, the fundamental role of seipin in LD homeostasis might explain both the impaired adipogenesis and the accelerated lipolysis reported in seipin deficiency.

\subsection{Other Mechanisms Contributing to the Adipocyte Loss}

Nevertheless, the function of seipin in mature adipocytes remains incompletely understood, and several elements need to be clarified. In 3T3-L1 adipocytes, we have shown that seipin is enriched at ER/LD contact sites under lipid loading and alternatively localizes at the ER/mitochondria contact sites also named MAMs [46]. Seipin interacts with MAM-associated calcium regulators, and seipin deficiency alters the ER to mitochondria $\mathrm{Ca}^{2+}$ flux, and this reduction is associated with a decrease in ATP production that is known to be dependent on mitochondrial $\mathrm{Ca}^{2+}$ levels [46]. These data are consistent with the work performed by Huang's lab that demonstrated in drosophila cells that seipin regulates ER $\mathrm{Ca}^{2+}$ handling $[63,64]$. Inducible-seipin deletion in mice leads to a progressive adipocyte loss associated with mitochondrial dysfunction. It has been previously shown that mitochondrial dysfunction can compromise adipocyte properties and finally cell survival [65]. 
Therefore, we can hypothesize that seipin function in controlling mitochondrial activity may explain, at least partially, the adipocyte loss caused by seipin deficiency (Figure 1B).

Finally, seipin deficiency in mature adipocytes induces the expression of ER stress markers including the pro-apoptotic C/EBP homologous protein (CHOP) transcription factor $[43,46]$. Therefore, ER stress might be one of the mechanisms involved in adipocyte loss. Seipin deficiency induces lipid remodeling, with an increase in several phospholipid species [43]. Changes in the PL ratio in the ER membrane might be an ER stress trigger [66]. Therefore, it would be useful to fractionate adipocytes and to assess the ER lipid composition. In addition, alterations in ER $\mathrm{Ca}^{2+}$ store and ATP deprivation might be other ER stress inducers. Further study should investigate if correcting the ER stress caused by seipin deficiency prevents the adipocyte loss.

\subsection{What about Brown Adipose Tissue?}

Whereas SKO mice or rats display at least a $90 \%$ decrease in WAT mass, the alteration of interscapular brow adipose tissue (BAT) mass is less severe, reaching a 30 to $55 \%$ reduction [37-40]. In vitro, seipin deficiency does not prevent brown adipocyte differentiation $[41,67]$. SKO mice can cope with cold acclimation, but they display a reduction in maximal thermogenic adaptation and are resistant to pro-thermogenic $B 3$ agonist treatment [41]. Specific seipin deficiency in BAT leads to an over-activation of the thermogenic/pro-catabolic program associated with BAT atrophy, mitochondrial dysfunction, and cell death $[67,68]$. Seipin deficiency in BAT leads to cold intolerance [68], supporting a cell-autonomous role for seipin in brown adipocytes.

\section{Metabolic Phenotype and Diabetic Complications}

\subsection{Adipose Tissue Loss Leads to Severe Insulin Resistance}

BSCL is defined in human patients by a nearly complete lack of adipose tissue associated with severe insulin resistance, which progresses to overt diabetes. SKO mice or rats are indeed severely lipodystrophic, and their metabolic phenotype has been studied [37-40]. All models display a 30 to $60 \%$ decrease in leptin levels associated with an increase in food intake [38,39]. Adiponectin levels are strongly affected [37-39], and this is associated with an impairment in glucose tolerance, insulin sensitivity, and a marked hyper-insulinemia. Hyperglycemia is visible in a random fed state only, not in the fasting condition [37-40]. Thus, SKO mice display metabolic inflexibility, and this is particularly severe during fasting, as they display a default in ketone bodies production, a drop in glycemia, and they are unable to maintain their body temperature [41].

Intriguingly, SKO mice are hypotriglyceridemic [37-39], but post-prandial TAG levels are elevated, supporting a default in lipid handling $[39,69]$. Our study showed that the low TAG levels might be due to an increase in TAG-rich lipoprotein uptake in the liver of SKO mice [38]. Of note, seipin-deficient rats are hypertriglyceridemic, suggesting that the rat could be a better model to study lipoproteins in the context of lipodystrophy [40].

In $\mathrm{SKO}$ mice, adipocyte-specific expression of seipin is sufficient to improve adipose tissue mass, leptin and adiponectin levels, insulin sensitivity, and glucose tolerance [70]. Consistently, adipose tissue transplantation improves post-prandial TAG, plasma leptin, glucose tolerance, insulin sensitivity, and insulin levels $[69,71]$. Leptin replacement also improves carbohydrate metabolism even though the effect is less marked than adipose tissue transplantation [71]. Taken together, these data support that lipodystrophy is the main trigger of carbohydrate metabolism alterations in SKO mice.

All models of adipocyte-specific deletion of seipin display lipodystrophy but variable metabolic responses. AP2-driven seipin deletion leads to insulin resistance and glucose intolerance in 6-month-old animals [43]. Inducible seipin deletion leads to glucose intolerance and insulin resistance 3 months after tamoxifen injection only $[45,46]$ if tamoxifen is reinjected every 3 months, avoiding the new differentiation of WAT adipocytes. In contrast, seipin deletion under the constitutive adipoQ promoter does not lead to glucose intolerance [42], but a $48 \mathrm{~h}$ high-fat diet (HFD) feeding is sufficient to trigger glucose intol- 
erance, suggesting that those mice are highly susceptible to metabolic complications [42] Altogether, the lack of adipose tissue is the major trigger of the metabolic complications associated with BSCL, and adipocyte seipin largely accounts for the development of carbohydrate metabolism abnormalities. However, as the metabolic complications appear later on or require an HFD trigger, this raises the question of the cell-autonomous function of seipin in metabolic tissues.

\subsection{Liver Steatosis Is Not Due to Seipin Loss in the Liver}

SKO mice and rats display an important hepatomegaly characterized by a massive liver steatosis [37-39] (Figure 2). To our knowledge, no report described the pathological state of the liver, i.e., simple steatosis or NAFLD, including fibrosis and inflammation. Liver lipid analysis revealed a 3 to 4 -fold increase in TAG and in DAG content [37,38,72]. DAG is well known to contribute to liver insulin resistance, and indeed, insulin signaling is blunted in SKO mice but improves by adipose tissue transplantation [69]. The re-expression of seipin in adipocytes only is sufficient to reduce liver steatosis in SKO mice [70], and consistently, the adipocyte-specific deletion of seipin leads to liver TAG accumulation [42,43]. Two mice models of restricted hepatic deletion of seipin have been generated, and their characterization did not reveal liver steatosis nor the associated metabolic complications [72,73]. Altogether, these data support that seipin deficiency in adipocytes leads to lipodystrophy that induces liver steatosis and liver insulin resistance. Animal and cellular studies support that there is no obvious cell-autonomous function for seipin in the hepatocyte. Therefore, seipin deficiency is a good model to study the effects of adipose tissue dysfunction on NAFLD development. Further studies should be performed to report more precisely the liver phenotype (fibrosis, inflammation) beyond lipid accumulation.

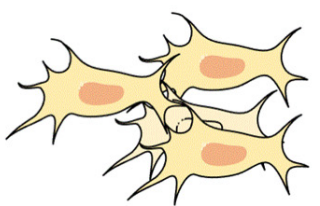

MSC

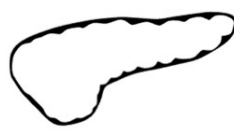

Pancreas

impaired insulin secretion

Cell autonomous?
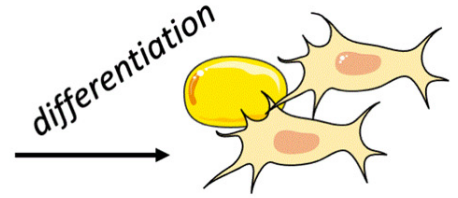

\pm Adipocyte

lipodystrophy

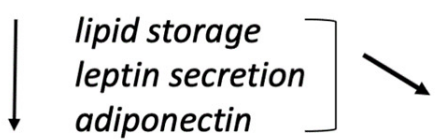

lipid Spillover

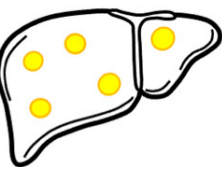

Liver

Steatosis

hyperglycemia

insulin-resistance

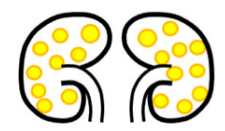

Kidneys

Lipid accumulation

AGE accumulation

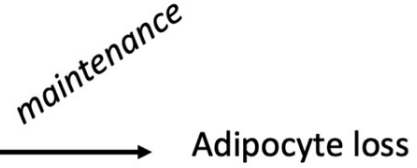

Adipocyte loss

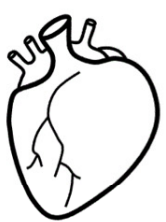

Heart

diastolic

dysfunction metabolic complications

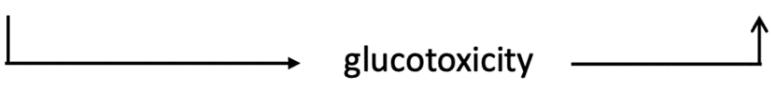

Figure 2. Physiopathology in seipin-deficient mice. Seipin deficiency leads to severe lipodystrophy that is the consequence of both adipogenesis impairment and alteration in mature adipocytes maintenance. 
The massive limitation in adipocyte storage promotes lipid ectopic accumulation in the liver and in the kidney. Liver steatosis leads to insulin resistance, which contributes to chronic hyperglycemia. Glucotoxic assault has been shown to contribute to kidney and heart dysfunction. Liver, kidney, and heart abnormalities are the consequences of adipose tissue dysfunction. On the other hand, pancreatic B-cells display impairment in insulin secretion that has been attributed to a cellautonomous function of seipin in this cell type. Mesenchymal stem cells (MSC), \pm adipocytes: mix of adipocytes \pm differentiated or apoptotic.

\subsection{A Valuable Model for Diabetic Cardiomyopathy}

Cardiomyopathy frequently occurs in BSCL2 patients and is characterized by concentric left ventricular hypertrophy (LVH) often associated with diastolic dysfunction but generally with preserved systolic function [74]. The phenotype is quite heterogeneous, and the cause is unclear. Most precisely, it is not clear whether this cardiomyopathy is associated with an ectopic lipid accumulation, as the results in the literature are contradictory [74-76]. SKO mice have been a useful model to characterize the cardiac phenotype associated with seipin deficiency. Hearts from SKO mice are heavier $[55,77,78]$ (Figure 2), and this was associated with a transcriptomic signature of an induction of the IGF1R/PI3K pathway [55]. Echography and MRI highlighted an LVH in SKO mice [55,77,78]. At the functional levels, the main issue is a diastolic dysfunction assessed by a decrease in the ratio between early (E) and late (atrial-A) ventricular filling velocity (E/A ratio) [77], an increase in isovolumetric relaxation time $[77,78]$, an alteration in the myocardial performance, and a modest but significant reduction in the ejection fraction. Altogether, this represents a valid model for diabetic cardiomyopathy. Lastly, transverse aortic constriction has a more severe effect in SKO mice [79]. However, the reports diverge on the potential cause for the cardiac dysfunction. We observed that the LVH is positively correlated with hyperglycemia, pointing out a role of glucose overload in the development of cardiac dysfunction. Thus, we assessed a chronic activation of the hexosamine pathway compatible with a glucotoxic mechanism [77]. Importantly, sodium-glucose co-transporter-2 (SGLT2) inhibitor treatment improves hyperglycemia and cardiac parameters suggesting that hyperglycemia resulting from insulin resistance is the main trigger of cardiac dysfunction in SKO mice. Of note, in this report, cardiomyopathy was detected in the absence of fibrosis, lipid abnormalities, or AGE accumulation. In contrast, another study proposed that seipin deficiency promotes excessive lipid catabolism in the heart that alters cardiac function [55]. The last report proposed that seipin deficiency induces neutrophil infiltration and severe fibrosis and an alteration in the phosphorylation of the contractile protein Titin that might change its functional properties [78]. Interestingly, these authors also showed that the heart-specific deletion of seipin does not lead to any cardiac dysfunction, supporting that cardiomyopathy is a consequence of lipodystrophy and the subsequent insulin resistance. Another group reported that in SKO mice, perivascular adipose tissue loses its anti-contractile properties [80]. This might also contribute to the diastolic dysfunction assessed in these animals. Altogether, SKO mice recapitulate the cardiomyopathy features and are unique tools to study the consequences of adipose tissue dysfunction on cardiac physiology. Importantly, lipid accumulation was not reported in any of these models, supporting that it was not the trigger of cardiac dysfunction.

\subsection{Other Metabolic Complications}

Kidney injury is a widely spread complication of diabetes. Lipodystrophic SKO mice display polyuria, increased albumin, creatinine, and ion excretion (Figure 2). These functional abnormalities were associated with an increase in glomerular and mesangial surface, along with lipid deposition, AGE accumulation, and hallmarks of oxidative stress, suggesting that lipotoxicity and glucotoxicty were both triggers of renal abnormalities. Of note, no sign of fibrosis or inflammation was detected. Importantly, leptin treatment and adipose tissue transplantation restore the physiological and morphological kidney properties, suggesting that kidney injury is a consequence of adipocyte dysfunction [71]. 
Muscle insulin resistance is a key contributor to chronic hyperglycemia in patients with type 2 diabetes. Insulin signaling appears to be normal [69] or slightly increased [81]. Muscles of SKO mice appear to accumulate less TAG and more glycogen, but the consequences on muscle physiology and metabolism are not clear yet. Seipin deletion in muscles does not alter muscle metabolism nor insulin signaling [81]. Therefore, seipin-deficient mice do not display major muscle insulin resistance nor lipid accumulation.

Intriguingly, whereas $\mathrm{Bscl}^{-/+}$heterozygous mice are not lipodystrophic nor insulin resistant, one study revealed a decrease in insulin secretion in isolated islet $B$-cells and in vivo in these animals [82]. Of note, neither lipotoxic nor glucotoxic hallmarks were reported in SKO mice islets (Figure 2). However, this does not translate into glucose intolerance. Specific BSCL2 deletion in the B-cell is needed to state whether seipin could play a cell-autonomous role in this key tissue for metabolic homeostasis. Given that calcium signaling plays a key role in glucose-stimulated insulin secretion in this cell type, studying the role of seipin in ER calcium handling would be particularly relevant in B-cells.

Finally, the only effect of seipin deficiency that appears independent of lipodystrophy in rodent models is the effect on brain function. As BSCL2 patients display mild mental retardation, several teams analyzed the central nervous system function in seipindeficient rodents. They reported deficits in learning and memory $[40,83]$ and in motor coordination [84] involving different mechanisms. These deficits have been observed in SKO mice and in neuronal-specific deficient (nSKO) mice but not in adipose-specific SKO mice, which indicates that they are not a consequence of lipodystrophy and illustrates the cell-autonomous role of seipin in nervous system. Both behavior and motor deficits are triggered by the reduction in PPAR $\gamma$ activity and interestingly, PPAR $\gamma$ agonists TZD alleviate the physiological alterations described above [83,84].

\section{Conclusions}

Twenty years ago, mutations in BSCL2 have been identified as the first cause for BSCL in human. Ten years ago, the first mouse model lacking seipin was published. In the last decade, an impressive number of studies intended to unravel how seipin deficiency leads to lipodystrophy and to cardiometabolic complications. At the cellular level, seipin is an ER anchored protein organized in multimers that are particularly enriched at ER/LD contact sites but also at ER/mitochondria contact sites. Seipin enrichment at one or the other ER contact site is nutritionally regulated. Seipin interacts with proteins involved in TAG synthesis, LD homeostasis, calcium handling, and mitochondrial function. Seipin deficiency impairs both adipocyte differentiation and mature adipocyte maintenance. The study of seipin function reinforced the importance of LD initiation-maintenance-expansion and illustrated how the homeostasis of this organelle is key in the adipocyte good health. Seipin also appears to have an unexpected role in $\mathrm{Ca}^{2+}$ exchange, and future work might help us to understand how $\mathrm{Ca}^{2+}$ signaling participates in adipocyte maintenance. Altogether, by studying one mysterious protein with no apparent functional domain, we are learning a lot about adipocyte biology.

Thus, seipin deficiency leads to severe lipodystrophy and cardiometabolic complications. Regarding diabetic cardiomyopathy, which remains a poorly understood complication, the data support that cardiac dysfunction takes place without lipid ectopic deposition, and one report supported a central harmful role of glucotoxicity. Therefore, SKO mice are a valuable model for studying diabetic cardiomyopathy pathophysiology and potentially to test new promising therapies.

Collectively, the studies discussed in this review support that liver steatosis, cardiomyopathy, and renal injury, classical diabetic complications, are all consequences of lipodystrophy. "It is not how fat you are, it is what you do with it that counts" [85], once said A Vidal-Puig. Seipin deficiency strikingly demonstrates that the lack of safe storage for lipids is a major threat for health. In contrast, adipose tissue transplantation and leptin treatment largely improve the SKO phenotype, demonstrating that increasing adipose tissue storage capacity deeply improves the metabolic health of these animals. Rare adipocyte 
dysfunctions such as in BSCL are key paradigm to unravel the pathways that control adipocyte good health. The knowledge gathered through the study of these pathologies may bring new strategies to maintain and improve adipose tissue expandability.

Author Contributions: J.M. and X.P. wrote the manuscript. All authors have read and agreed to the published version of the manuscript.

Funding: This study has been granted from the FFRD (sponsored by Fédération Française des Diabétiques, Abbott, AstraZeneca, Eli Lilly, Merck Sharp \& Dohme and Novo Nordisk) and the French National Research Agency (ANR-21-CE14-0024 MAMA).

Institutional Review Board Statement: Not applicable.

Informed Consent Statement: Not applicable.

Data Availability Statement: Not applicable.

Conflicts of Interest: The authors declare no conflict of interest.

\section{References}

1. Kivimäki, M.; Kuosma, E.; Ferrie, J.E.; Luukkonen, R.; Nyberg, S.T.; Alfredsson, L.; Batty, G.D.; Brunner, E.J.; Fransson, E.; Goldberg, M.; et al. Overweight, obesity, and risk of cardiometabolic multimorbidity: Pooled analysis of individual-level data for 120813 adults from 16 cohort studies from the USA and Europe. Lancet Public Health 2017, 2, e277-e285. [CrossRef]

2. Virtue, S.; Vidal-Puig, A. Adipose tissue expandability, lipotoxicity and the Metabolic Syndrome-An allostatic perspective. Biochim. Biophys. Acta 2010, 1801, 338-349. [CrossRef] [PubMed]

3. Savage, D.B.; Petersen, K.F.; Shulman, G.I. Disordered lipid metabolism and the pathogenesis of insulin resistance. Physiol. Rev. 2007, 87, 507-520. [CrossRef] [PubMed]

4. Cuthbertson, D.J.; Steele, T.; Wilding, J.P.; Halford, J.C.; Harrold, J.A.; Hamer, M.; Karpe, F. What have human experimental overfeeding studies taught us about adipose tissue expansion and susceptibility to obesity and metabolic complications? Int. J. Obes. 2017, 41, 853-865. [CrossRef] [PubMed]

5. Yaghootkar, H.; Lotta, L.A.; Tyrrell, J.; Smit, R.A.J.; Jones, S.E.; Donnelly, L.; Beaumont, R.; Campbell, A.; Tuke, M.A.; Hayward, C.; et al. Genetic Evidence for a Link Between Favorable Adiposity and Lower Risk of Type 2 Diabetes, Hypertension, and Heart Disease. Diabetes 2016, 65, 2448-2460. [CrossRef]

6. Mann, J.P.; Savage, D.B. What lipodystrophies teach us about the metabolic syndrome. J. Clin. Investig. 2019, $129,4009-4021$. [CrossRef]

7. Lim, K.; Haider, A.; Adams, C.; Sleigh, A.; Savage, D.B. Lipodistrophy: A paradigm for understanding the consequences of "overloading" adipose tissue. Physiol. Rev. 2021, 101, 907-993. [CrossRef]

8. Garg, A. Clinical review\#: Lipodystrophies: Genetic and acquired body fat disorders. J. Clin. Endocrinol. Metab. 2011, 96, 3313-3325. [CrossRef]

9. Berardinelli, W. An undiagnosed endocrinometabolic syndrome: Report of two cases. J. Clin. Endocrinol. Metab. 1954, 14, 193-204. [CrossRef]

10. Seip, M. Lipoatrophy and gigantism with associated endocrine manifestations: A new diencephalic syndrome? Acta Paediatr. Scand. 1959, 48, 555-574. [CrossRef]

11. Agarwal, A.K.; Arioglu, E.; De Almeida, S.; Akkoc, N.; Taylor, S.I.; Bowcock, A.M.; Barnes, R.I.; Garg, A. AGPAT2 is mutated in congenital generalized lipodystrophy linked to chromosome 9q34. Nat. Genet. 2002, 31, 21-23. [CrossRef]

12. Magré, J.; Delépine, M.; Khallouf, E.; Gedde-Dahl, T., Jr.; Van Maldergem, L.; Sobel, E.; Papp, J.; Meier, M.; Mégarbané, A.; Bachy, A.; et al. Identification of the gene altered in Berardinelli-Seip congenital lipodystrophy on chromosome 11q13. Nat. Genet. 2001, 28, 365-370. [CrossRef]

13. Kim, C.A.; Delepine, M.; Boutet, E.; El Mourabit, H.; Le Lay, S.; Meier, M.; Nemani, M.; Bridel, E.; Leite, C.C.; Bertola, D.R.; et al. Association of a homozygous nonsense caveolin-1 mutation with Berardinelli-Seip congenital lipodystrophy. J. Clin. Endocrinol. Metab. 2008, 93, 1129-1134. [CrossRef] [PubMed]

14. Hayashi, Y.K.; Matsuda, C.; Ogawa, M.; Goto, K.; Tominaga, K.; Mitsuhashi, S.; Park, Y.E.; Nonaka, I.; Hino-Fukuyo, N.; Haginoya, K.; et al. Human PTRF mutations cause secondary deficiency of caveolins resulting in muscular dystrophy with generalized lipodystrophy. J. Clin. Investig. 2009, 119, 2623-2633. [CrossRef]

15. Gomes, K.B.; Salles Moura Fernandes, A.P.; Souza Ferreira, A.C.; Pardini, H.; Garg, A.; Magré, J.; Pardini, V.C. Mutations in the Seipin gene and AGPAT2 clustering in consanguineous families with Berardinelli-Seip congenital lipodystrophy from two separate geographical regions in Brazil. J. Clin. Endocrinol. Metab. 2004, 89, 357-361. [CrossRef] [PubMed]

16. Araujo-Vilar, D.; Santini, F. Diagnosis and treatment of lipodystrophy: A step-by-step approach. J. Endocrinol. Investig. 2019, 42, 61-73. [CrossRef] [PubMed] 
17. Craveiro Sarmento, A.S.; Ferreira, L.C.; Lima, J.G.; de Azevedo Medeiros, L.B.; Barbosa Cunha, P.T.; Agnez-Lima, L.F.; Galvao Ururahy, M.A.; de Melo Campos, J.T.A. The worldwide mutational landscape of Berardinelli-Seip congenital lipodystrophy. Mutat. Res. Rev. Mutat. Res. 2019, 781, 30-52. [CrossRef] [PubMed]

18. Agarwal, A.K.; Simha, V.; Arioglu Oral, E.; Moran, S.A.; Gorden, P.; O’Rahilly, S.; Zaidi, Z.; Gurakan, F.; Arslanian, S.A.; Klar, A.; et al. Phenotypic and Genetic heterogeneity in Congenital Generalized Lipodystrophy. J. Clin. Endocrinol. Metab. 2003, 88, 4840-4847. [CrossRef]

19. Van Maldergem, L.; Magré, J.; Gedde-DahlJr, T.; Khallouf, E.; Delépine, M.; Trygstad, O.; Seemova, E.; Stephenson, T.; Albott, C.S.; Bonnici, F.; et al. Genotype-Phenotype Relationships in Berardinelli-Seip Congenital Lipodystrophy. J. Med. Genet. 2002, 39, 722-733. [CrossRef]

20. Agarwal, A.K.; Garg, A. Congenital generalized lipodystrophy: Significance of triglyceride biosynthetic pathways. Trends Endocrinol. Metab. 2003, 14, 214-221. [CrossRef]

21. Patni, N.; Garg, A. Congenital generalized lipodystrophies-new insights into metabolic dysfunction. Nat. Rev. Endocrinol. 2015, 11, 522-534. [CrossRef]

22. Robbins, A.L.; Savage, D.B. The genetics of lipid storage and human lipodystrophies. Trends Mol. Med. 2015, 21, 433-438. [CrossRef]

23. Szymanski, K.M.; Binns, D.; Bartz, R.; Grishin, N.V.; Li, W.P.; Agarwal, A.K.; Garg, A.; Anderson, R.G.; Goodman, J.M. The lipodystrophy protein seipin is found at endoplasmic reticulum lipid droplet junctions and is important for droplet morphology. Proc. Natl. Acad. Sci. USA 2007, 104, 20890-20895. [CrossRef]

24. Fei, W.; Shui, G.; Gaeta, B.; Du, X.; Kuerschner, L.; Li, P.; Brown, A.J.; Wenk, M.R.; Parton, R.G.; Yang, H. Fld1p, a functional homologue of human seipin, regulates the size of lipid droplets in yeast. J. Cell Biol. 2008, 180, 473-482. [CrossRef] [PubMed]

25. Fei, W.; Shui, G.; Zhang, Y.; Krahmer, N.; Ferguson, C.; Kapterian, T.S.; Lin, R.C.; Dawes, I.W.; Brown, A.J.; Li, P.; et al. A role for phosphatidic Acid in the formation of "supersized" lipid droplets. PLoS Genet. 2011, 7, e1002201. [CrossRef]

26. Boutet, E.; El Mourabit, H.; Prot, M.; Nemani, M.; Khallouf, E.; Colard, O.; Maurice, M.; Durand-Schneider, A.M.; Chretien, Y.; Gres, S.; et al. Seipin deficiency alters fatty acid Delta9 desaturation and lipid droplet formation in Berardinelli-Seip congenital lipodystrophy. Biochimie 2009, 91, 796-803. [CrossRef]

27. Lundin, C.; Nordstrom, R.; Wagner, K.; Windpassinger, C.; Andersson, H.; von Heijne, G.; Nilsson, I. Membrane topology of the human seipin protein. FEBS Lett. 2006, 580, 2281-2284. [CrossRef]

28. Wee, K.; Yang, W.; Sugii, S.; Han, W. Towards a mechanistic understanding of lipodystrophy and seipin functions. Biosci. Rep. 2014, 34, e00141. [CrossRef]

29. Binns, D.; Lee, S.; Hilton, C.L.; Jiang, Q.X.; Goodman, J.M. Seipin is a discrete homooligomer. Biochemistry 2010, 49, 10747-10755. [CrossRef] [PubMed]

30. Yan, R.; Qian, H.; Lukmantara, I.; Gao, M.; Du, X.; Yan, N.; Yang, H. Human SEIPIN Binds Anionic Phospholipids. Dev. Cell 2018, 47, 248-256. [CrossRef] [PubMed]

31. Sui, X.; Arlt, H.; Brock, K.P.; Lai, Z.W.; DiMaio, F.; Marks, D.S.; Liao, M.; Farese, R.V., Jr.; Walther, T.C. Cryo-electron microscopy structure of the lipid droplet-formation protein seipin. J. Cell Biol. 2018, 217, 4080-4091. [CrossRef]

32. Talukder, M.U.; Sim, M.F.M.; O'Rahilly, S.; Edwardson, J.M.; Rochford, J.J. Seipin oligomers can interact directly with AGPAT2 and lipin 1, physically scaffolding critical regulators of adipogenesis. Mol. Metab. 2015, 4, 199-209. [CrossRef]

33. Prasanna, X.; Salo, V.T.; Li, S.; Ven, K.; Vihinen, H.; Jokitalo, E.; Vattulainen, I.; Ikonen, E. Seipin traps triacylglycerols to facilitate their nanoscale clustering in the endoplasmic reticulum membrane. PLoS Biol. 2021, 19, e3000998. [CrossRef]

34. Zoni, V.; Khaddaj, R.; Lukmantara, I.; Shinoda, W.; Yang, H.; Schneiter, R.; Vanni, S. Seipin accumulates and traps diacylglycerols and triglycerides in its ring-like structure. Proc. Natl. Acad. Sci. USA 2021, 118, e2017205118. [CrossRef]

35. Payne, V.A.; Grimsey, N.; Tuthill, A.; Virtue, S.; Gray, S.L.; Dalla Nora, E.; Semple, R.K.; O'Rahilly, S.; Rochford, J.J. The human lipodystrophy gene BSCL2/seipin may be essential for normal adipocyte differentiation. Diabetes 2008, 57, 2055-2060. [CrossRef] [PubMed]

36. Chen, W.; Yechoor, V.K.; Chang, B.H.; Li, M.V.; March, K.L.; Chan, L. The human lipodystrophy gene product Berardinelli-Seip congenital lipodystrophy 2/seipin plays a key role in adipocyte differentiation. Endocrinology 2009, 150, 4552-4561. [CrossRef]

37. Cui, X.; Wang, Y.; Tang, Y.; Liu, Y.; Zhao, L.; Deng, J.; Xu, G.; Peng, X.; Ju, S.; Liu, G.; et al. Seipin ablation in mice results in severe generalized lipodystrophy. Hum. Mol. Genet. 2011, 20, 3022-3030. [CrossRef]

38. Prieur, X.; Dollet, L.; Takahashi, M.; Nemani, M.; Pillot, B.; Le May, C.; Mounier, C.; Takigawa-Imamura, H.; Zelenika, D.; Matsuda, F.; et al. Thiazolidinediones partially reverse the metabolic disturbances observed in Bscl2/seipin-deficient mice. Diabetologia 2013, 56, 1813-1825. [CrossRef] [PubMed]

39. Chen, W.; Chang, B.; Saha, P.; Hartig, S.M.; Li, L.; Reddy, V.T.; Yang, Y.; Yechoor, V.; Mancini, M.A.; Chan, L. Berardinelli-seip congenital lipodystrophy 2 /seipin is a cell-autonomous regulator of lipolysis essential for adipocyte differentiation. Mol. Cell. Biol. 2012, 32, 1099-1111. [CrossRef]

40. Ebihara, C.; Ebihara, K.; Aizawa-Abe, M.; Mashimo, T.; Tomita, T.; Zhao, M.; Gumbilai, V.; Kusakabe, T.; Yamamoto, Y.; Aotani, D.; et al. Seipin is necessary for normal brain development and spermatogenesis in addition to adipogenesis. Hum. Mol. Genet. 2015, 24, 4238-4249. [CrossRef] [PubMed] 
41. Dollet, L.; Magré, J.; Joubert, M.; Le May, C.; Ayer, A.; Arnaud, L.; Pecqueur, C.; Blouin, V.; Cariou, B.; Prieur, X. Seipin deficiency alters brown adipose tissue thermogenesis and insulin sensitivity in a non-cell autonomous mode. Sci. Rep. 2016, 6, 35487. [CrossRef]

42. Mcilroy, G.D.; Suchacki, K.; Roelofs, A.J.; Yang, W.; Fu, Y.; Bai, B.; Wallace, R.J.; De Bari, C.; Cawthorn, W.P.; Han, W.; et al. Adipose specific disruption of seipin causes early-onset generalised lipodystrophy and altered fuel utilisation without severe metabolic disease. Mol. Metab. 2018, 10, 55-65. [CrossRef]

43. Liu, L.; Jiang, Q.; Wang, X.; Zhang, Y.; Lin, R.C.; Lam, S.M.; Shui, G.; Zhou, L.; Li, P.; Wang, Y.; et al. Adipose-specific knockout of Seipin/Bscl2 results in progressive lipodystrophy. Diabetes 2014, 63, 2320-2331. [CrossRef]

44. Jeffery, E.; Berry, R.; Church, C.D.; Yu, S.; Shook, B.A.; Horsley, V.; Rosen, E.D.; Rodeheffer, M.S. Characterization of Cre recombinase models for the study of adipose tissue. Adipocyte 2014, 3, 206-211. [CrossRef] [PubMed]

45. Zhou, H.; Lei, X.; Benson, T.; Mintz, J.; Xu, X.; Harris, R.B.; Weintraub, N.L.; Wang, X.; Chen, W. Berardinelli-Seip congenital lipodystrophy 2 regulates adipocyte lipolysis, browning, and energy balance in adult animals. J. Lipid Res. 2015, 56, 1912-1925. [CrossRef] [PubMed]

46. Combot, Y.; Salo, V.T.; Chadeuf, G.; Hölttä, M.; Ven, K.; Pulli, I.; Ducheix, S.; Pecqueur, C.; Renoult, O.; Lak, B.; et al. Seipin localizes at endoplasmic reticulum-mitochondria contact sites to control mitochondrial calcium import and metabolism in adipocytes. Cell Rep. 2021, 38, 110213. [CrossRef]

47. Chaves, C.; Chaves, M.; Anselmo, J.; Cesar, R. Successful long-term use of pioglitazone in Berardinelli-Seip lipodystrophyassociated diabetes. Endocrinol. Diabetes Metab. Case Rep. 2021, 2021. [CrossRef] [PubMed]

48. Fei, W.; Du, X.; Yang, H. Seipin, adipogenesis and lipid droplets. Trends Endocrinol. Metab. 2011, 22, 204-210. [CrossRef]

49. Pagac, M.; Cooper, D.E.; Qi, Y.; Lukmantara, I.E.; Mak, H.Y.; Wu, Z.; Tian, Y.; Liu, Z.; Lei, M.; Du, X.; et al. SEIPIN Regulates Lipid Droplet Expansion and Adipocyte Development by Modulating the Activity of Glycerol-3-phosphate Acyltransferase. Cell Rep. 2016, 17, 1546-1559. [CrossRef]

50. Gao, M.; Liu, L.; Wang, X.; Mak, H.Y.; Liu, G.; Yang, H. GPAT3 deficiency alleviates insulin resistance and hepatic steatosis in a mouse model of severe congenital generalized lipodystrophy. Hum. Mol. Genet. 2020, 29, 432-443. [CrossRef]

51. Sim, M.F.M.; Dennis, R.J.; Aubry, E.M.; Ramanathan, N.; Sembongi, H.; Saudek, V.; Ito, D.; O’Rahilly, S.; Siniossoglou, S.; Rochford, J.J. The human lipodystrophy protein seipin is an ER membrane adaptor for the adipogenic PA phosphatase lipin 1. Mol. Metab. 2012, 2, 38-46. [CrossRef]

52. Grimsey, N.; Han, G.S.; O'Hara, L.; Rochford, J.J.; Carman, G.M.; Siniossoglou, S. Temporal and spatial regulation of the phosphatidate phosphatases lipin 1 and 2. J. Biol. Chem. 2008, 283, 29166-29174. [CrossRef] [PubMed]

53. Subauste, A.R.; Das, A.K.; Li, X.; Elliott, B.G.; Elliot, B.; Evans, C.; El Azzouny, M.; Treutelaar, M.; Oral, E.; Leff, T.; et al. Alterations in lipid signaling underlie lipodystrophy secondary to AGPAT2 mutations. Diabetes 2012, 61, 2922-2931. [CrossRef]

54. Gale, S.E.; Frolov, A.; Han, X.; Bickel, P.E.; Cao, L.; Bowcock, A.; Schaffer, J.E.; Ory, D.S. A regulatory role for 1-acylglycerol-3phosphate-O-acyltransferase 2 in adipocyte differentiation. J. Biol. Chem. 2006, 281, 11082-11089. [CrossRef]

55. Zhou, H.; Lei, X.; Yan, Y.; Lydic, T.; Li, J.; Weintraub, N.L.; Su, H.; Chen, W. Targeting ATGL to rescue BSCL2 lipodystrophy and its associated cardiomyopathy. JCI Insight 2019, 4, e129781. [CrossRef]

56. Chen, W.; Zhou, H.; Liu, S.; Fhaner, C.J.; Gross, B.C.; Lydic, T.A.; Reid, G.E. Altered lipid metabolism in residual white adipose tissues of Bscl2 deficient mice. PLoS ONE 2013, 8, e82526. [CrossRef] [PubMed]

57. Cartwright, B.R.; Binns, D.D.; Hilton, C.L.; Han, S.; Gao, Q.; Goodman, J.M. Seipin performs dissectible functions in promoting lipid droplet biogenesis and regulating droplet morphology. Mol. Biol. Cell 2015, 26, 726-739. [CrossRef]

58. Wang, H.; Becuwe, M.; Housden, B.E.; Chitraju, C.; Porras, A.J.; Graham, M.M.; Liu, X.N.; Thiam, A.R.; Savage, D.B.; Agarwal, A.K.; et al. Seipin is required for converting nascent to mature lipid droplets. eLife 2016, 5, e16582. [CrossRef] [PubMed]

59. Salo, V.T.; Belevich, I.; Li, S.; Karhinen, L.; Vihinen, H.; Vigouroux, C.; Magré, J.; Thiele, C.; Hölttä-Vuori, M.; Jokitalo, E.; et al. Seipin regulates ER-lipid droplet contacts and cargo delivery. EMBO J. 2016, 35, 2699-2716. [CrossRef] [PubMed]

60. Wang, S.; Idrissi, F.Z.; Hermansson, M.; Grippa, A.; Ejsing, C.S.; Carvalho, P. Seipin and the membrane-shaping protein Pex30 cooperate in organelle budding from the endoplasmic reticulum. Nat. Commun. 2018, 9, 2939. [CrossRef]

61. Salo, V.T.; Li, S.; Vihinen, H.; Holtta-Vuori, M.; Szkalisity, A.; Horvath, P.; Belevich, I.; Peranen, J.; Thiele, C.; Somerharju, P.; et al. Seipin Facilitates Triglyceride Flow to Lipid Droplet and Counteracts Droplet Ripening via Endoplasmic Reticulum Contact. Dev. Cell 2019, 50, 478-493. [CrossRef]

62. Rao, M.J.; Goodman, J.M. Seipin: Harvesting fat and keeping adipocytes healthy. Trends Cell Biol. 2021, 31, 912-923. [CrossRef]

63. Ding, L.; Yang, X.; Tian, H.; Liang, J.; Zhang, F.; Wang, G.; Wang, Y.; Ding, M.; Shui, G.; Huang, X. Seipin regulates lipid homeostasis by ensuring calcium-dependent mitochondrial metabolism. EMBO J. 2018, 37, e97572. [CrossRef] [PubMed]

64. Bi, J.; Wang, W.; Liu, Z.; Huang, X.; Jiang, Q.; Liu, G.; Wang, Y.; Huang, X. Seipin promotes adipose tissue fat storage through the ER Ca ${ }^{2+}$-ATPase SERCA. Cell Metab. 2014, 19, 861-871. [CrossRef]

65. Kusminski, C.M.; Scherer, P.E. Mitochondrial dysfunction in white adipose tissue. Trends Endocrinol. Metab. 2012, 23, 435-443. [CrossRef] [PubMed]

66. Fu, S.; Yang, L.; Li, P.; Hofmann, O.; Dicker, L.; Hide, W.; Lin, X.; Watkins, S.M.; Ivanov, A.R.; Hotamisligil, G.S. Aberrant lipid metabolism disrupts calcium homeostasis causing liver endoplasmic reticulum stress in obesity. Nature 2011, 473, 528-531. [CrossRef] [PubMed] 
67. Zhou, H.; Black, S.M.; Benson, T.W.; Weintraub, N.L.; Chen, W. Berardinelli-Seip Congenital Lipodystrophy 2/Seipin Is Not Required for Brown Adipogenesis but Regulates Brown Adipose Tissue Development and Function. Mol. Cell. Biol. 2016, 36, 2027-2038. [CrossRef] [PubMed]

68. Zhou, H.; Xu, C.; Lee, H.; Yoon, Y.; Chen, W. Berardinelli-Seip congenital lipodystrophy 2/SEIPIN determines brown adipose tissue maintenance and thermogenic programing. Mol. Metab. 2020, 36, 100971. [CrossRef]

69. Wang, H.; Xu, P.F.; Li, J.Y.; Liu, X.J.; Wu, X.Y.; Xu, F.; Xie, B.C.; Huang, X.M.; Zhou, Z.H.; Kayoumu, A.; et al. Adipose tissue transplantation ameliorates lipodystrophy-associated metabolic disorders in seipin-deficient mice. Am. J. Physiol. Endocrinol. Metab. 2019, 316, E54-E62. [CrossRef]

70. Gao, M.; Wang, M.; Guo, X.; Qiu, X.; Liu, L.; Liao, J.; Liu, J.; Lu, G.; Wang, Y.; Liu, G. Expression of seipin in adipose tissue rescues lipodystrophy, hepatic steatosis and insulin resistance in seipin null mice. Biochem. Biophys. Res. Commun. 2015, 460, 143-150. [CrossRef]

71. Liu, X.J.; Wu, X.Y.; Wang, H.; Wang, S.X.; Kong, W.; Zhang, L.; Liu, G.; Huang, W. Renal injury in Seipin-deficient lipodystrophic mice and its reversal by adipose tissue transplantation or leptin administration alone: Adipose tissue-kidney crosstalk. FASEB J. 2018, 32, 5550-5562. [CrossRef] [PubMed]

72. Chen, W.; Zhou, H.; Saha, P.; Li, L.; Chan, L. Molecular mechanisms underlying fasting modulated liver insulin sensitivity and metabolism in male lipodystrophic Bscl2/Seipin-deficient mice. Endocrinology 2014, 155, 4215-4225. [CrossRef]

73. McIlroy, G.D.; Mitchell, S.E.; Han, W.; Delibegovic, M.; Rochford, J.J. Ablation of Bscl2/seipin in hepatocytes does not cause metabolic dysfunction in congenital generalised lipodystrophy. Dis. Model. Mech. 2020, 13, dmm042655. [CrossRef]

74. Lupsa, B.C.; Sachdev, V.; Lungu, A.O.; Rosing, D.R.; Gorden, P. Cardiomyopathy in congenital and acquired generalized lipodystrophy: A clinical assessment. Medicine 2010, 89, 245-250. [CrossRef] [PubMed]

75. Nelson, M.D.; Victor, R.G.; Szczepaniak, E.W.; Simha, V.; Garg, A.; Szczepaniak, L.S. Cardiac steatosis and left ventricular hypertrophy in patients with generalized lipodystrophy as determined by magnetic resonance spectroscopy and imaging. Am. J. Cardiol. 2013, 112, 1019-1024. [CrossRef] [PubMed]

76. Rheuban, K.S.; Blizzard, R.M.; Parker, M.A.; Carter, T.; Wilson, T.; Gutgesell, H.P. Hypertrophic cardiomyopathy in total lipodystrophy. J. Pediatr. 1986, 109, 301-312. [CrossRef]

77. Joubert, M.; Jagu, B.; Montaigne, D.; Marechal, X.; Tesse, A.; Ayer, A.; Dollet, L.; Le May, C.; Toumaniantz, G.; Manrique, A.; et al. The Sodium-Glucose Cotransporter 2 Inhibitor Dapagliflozin Prevents Cardiomyopathy in a Diabetic Lipodystrophic Mouse Model. Diabetes 2017, 66, 1030-1040. [CrossRef] [PubMed]

78. Bai, B.; Yang, W.; Fu, Y.; Foon, H.L.; Tay, W.T.; Yang, K.; Luo, C.; Gunaratne, J.; Lee, P.; Zile, M.R.; et al. Seipin Knockout Mice Develop Heart Failure With Preserved Ejection Fraction. JACC Basic Transl. Sci. 2019, 4, 924-937. [CrossRef]

79. Wu, X.; Liu, X.; Wang, H.; Zhou, Z.; Yang, C.; Li, Z.; Zhang, Y.; Shi, X.; Zhang, L.; Wang, Y.; et al. Seipin Deficiency Accelerates Heart Failure Due to Calcium Handling Abnormalities and Endoplasmic Reticulum Stress in Mice. Front. Cardiovasc. Med. 2021, 8, 644128. [CrossRef] [PubMed]

80. Wang, M.; Xing, J.; Liu, M.; Gao, M.; Liu, Y.; Li, X.; Hu, L.; Zhao, X.; Liao, J.; Liu, G.; et al. Deletion of Seipin Attenuates Vascular Function and the Anticontractile Effect of Perivascular Adipose Tissue. Front. Cardiovasc. Med. 2021, 8, 706924. [CrossRef] [PubMed]

81. Xu, W.; Zhou, H.; Xuan, H.; Saha, P.; Wang, G.; Chen, W. Novel metabolic disorders in skeletal muscle of Lipodystrophic Bscl2/Seipin deficient mice. Mol. Cell. Endocrinol. 2019, 482, 1-10. [CrossRef]

82. Xiong, J.; Sun, P.; Wang, Y.; Hua, X.; Song, W.; Wang, Y.; Wu, J.; Yu, W.; Liu, G.; Chen, L. Heterozygous deletion of Seipin in islet beta cells of male mice has an impact on insulin synthesis and secretion through reduced PPARgamma expression. Diabetologia 2020, 63, 338-350. [CrossRef] [PubMed]

83. Zhou, L.; Chen, T.; Li, G.; Wu, C.; Wang, C.; Li, L.; Sha, S.; Chen, L.; Liu, G.; Chen, L. Activation of PPARgamma Ameliorates Spatial Cognitive Deficits through Restoring Expression of AMPA Receptors in Seipin Knock-Out Mice. J. Neurosci. 2016, 36 , 1242-1253. [CrossRef]

84. Wang, L.; Hong, J.; Wu, Y.; Liu, G.; Yu, W.; Chen, L. Seipin deficiency in mice causes loss of dopaminergic neurons via aggregation and phosphorylation of alpha-synuclein and neuroinflammation. Cell Death Dis. 2018, 9, 440. [CrossRef] [PubMed]

85. Virtue, S.; Vidal-Puig, A. It's not how fat you are, it's what you do with it that counts. PLoS Biol. 2008, 6, e237. [CrossRef] [PubMed] 